\title{
Características distintivas del desarrollo de las nanotecnologías en América Latina
}

\author{
OULLLERNO FOLADORI" \\ SANTIAGO FIEUEROA* \\ ZÁYAGO-LAU EDGARD \\ NOELA INUERNIZZ1
}

\section{Resumen}

Este trabajo analiza el desarrollo de las políticas públicas de nanotecnología en Brasil, México y Argentina. Dos características distintivas fueron detectadas, aunque con diferentes grados de presencia en cada país. La primera es la creación de centros de excelencia científica integrada a la industria, con el propósito de incrementar la competencia internacional. La segunda característica es la falta de atención a los aspectos sociales incorporados a esta revolución tecnológica, tales como la calificación de la fuerza de trabajo y la protección de consumidores y trabajadores frente a los potenciales riesgos, así como promover la participación de organizaciones sociales en las decisiones de políticas públicas.

Palabras clave: Nanotecnología. América Latina. Políticas públicas.

\footnotetext{
* Universidad Autónoma de Zacatecas, México \& ReLANS (Red Latinoamericana de Nanotecnología y Sociedad). Parcialmente financiado por la beca UC-Mexus-Conacyt CN 10-420. (México). E-mail: gfoladori@gmail.com

** ReLANS, miembro postdoctoral en el Laboratorio Sincrotrón de Grenoble, (Francia). E-mail: figueroa@inifta.unlp.edu.ar

*** Universidad Autónoma de Zacatecas, México \& ReLANS (Red Latinoamericana de Nanotecnología y Sociedad). Parcialmente financiado por el programa PROMEP UAZ-PTC-122. (México). E-mail: zayagolau@gmail.com.

${ }^{* * * *}$ Universidad Federal de Paraná, Brasil \& ReLANS. Miembro del Programa de Ciência, Tecnología e Innovación. Centro Internacional para Académicos Woodrow Wilson. (Brasil). Email: noela.invernizzi@gmail.com
} 


\section{Distinctive features of the development of nanotechnologies in Latin America}

\section{Abstract}

This paper analyses the development of public policies on nanotechnology (NT) in Brazil, Mexico and Argentina. Two main distinctive features were detected, although with different levels of presence in each country.

The first feature is the creation of centers of scientific excellence integrated with industry, with the aim of increasing international competitiveness. The second feature is the lack of attention to social issues involved in this technological revolution, such as educating the workforce, protecting consumers and workers from potential risks, and promoting the participation of social organizations into public policy decisions.

Key words: Nanotechnology. Latin America. Public policies.

\section{Introducción}

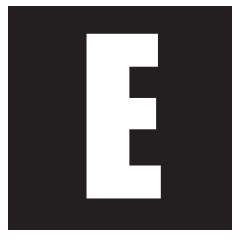

ste trabajo analiza el desarrollo de las políticas públicas en nanotecnología (NT) en Brasil, México y Argentina. Estos son los países de América Latina donde el desarrollo de la NT está más avanzado. Aunque hay diferencias entre ellos, debidas principalmente a su distinta integración económica al mercado mundial y las políticas de cada país respecto al balance entre el mercado externo e interno, se pueden identificar algunas características comunes del desarrollo de la NT. Dos rasgos principales se han detectado, aunque con diferente nivel de presencia en cada país.

El primer rasgo es la tendencia a la creación de centros de excelencia científica integrados a la industria, con el propósito de mejorar la competitividad internacional. Bajo un contexto de economías abiertas, la política de Ciencia y Tecnología (C\&T) priorizó la ciencia aplicada, la 
evaluación de la actividad científica según estándares internacionales, y la incorporación de la investigación nacional a temas de vanguardia en la escena mundial. Esta estrategia requirió inversiones en infraestructura y equipamiento para crear centros de investigación competitivos con los de los países desarrollados.

El segundo rasgo es la falta de atención a las cuestiones sociales, tales como la calificación de la fuerza de trabajo, la protección de los consumidores y los trabajadores contra los riesgos potenciales, y la promoción de la participación de las organizaciones sociales en las decisiones de política pública.

El artículo está organizado de la siguiente manera: en la primera sección se revisan las recomendaciones de las instituciones internacionales que impulsaron a la NT como un área estratégica en C\&T en los países de América Latina. En la segunda sección se proporciona un resumen de cómo la NT se incorporó a las políticas de C\&T en los tres países, y cómo los objetivos de esas políticas se relacionan con un contexto de economías cada vez más abiertas. En la tercera sección se analiza la mejora de la infraestructura de laboratorios, un paso fundamental en los esfuerzos para promover la investigación de clase mundial en NT. En la cuarta sección se plantea la estrategia de desarrollo de NT que subyace en las políticas públicas basadas en la asociación de empresas privadas con centros de investigación públicos. En la quinta sección se evalúan los principales elementos que caracterizan a las políticas de NT en estos países, así como los aspectos clave que deben ser considerados pero no están incluidos en las políticas.

Influencia de las instituciones internacionales sobre las políticas de nanotecnología en América Latina

Los tres países más avanzados en Investigación y Desarrollo (I\&D) en NT en América Latina son Brasil, México y Argentina. Entre 2000 y 
2007 Brasil produjo 5254 publicaciones científicas en NT; México 2261 y Argentina 1376. En términos de patentes, y entre 2000 y 2006, Brasil registró la mayoría en América Latina con 89, mientras que México registró 28 y Argentina 12 (OIA-CTS, 2008). El siguiente análisis se basa principalmente en estos países.

La I\&D en NT, aunque bajo el nombre de partículas ultrafinas, se inició en los años 80 y 90, como ocurrió en los países desarrollados, mucho antes de las políticas públicas destinadas a su fomento. Sin embargo, cuando Estados Unidos lanzó la Iniciativa Nacional de Nanotecnología en el año 2000, incentivó el desarrollo de estas ciencias y tecnologías asociadas en el resto del mundo. Organizaciones internacionales como el Banco Mundial (BM) han estado promoviendo la estrategia de desarrollo conocida como "economía del conocimiento" desde la década de 1990, en la que el desarrollo está estrechamente relacionado con la innovación y las altas tecnologías. La NT se incorporó rápidamente como un área estratégica de I\&D en ese contexto teórico. En 1991, un reporte del BM hizo hincapié en la importancia de invertir en activos intangibles para promover el desarrollo (Banco Mundial, 1991, p. 33-35). El BM considera que una economía del conocimiento es:

... una en la que los activos de conocimiento son deliberadamente más importantes que los activos de capital y de mano de obra, y donde la cantidad y sofisticación del conocimiento que impregna la actividad económica y social alcanza niveles muy altos (Banco Mundial, 2007, p. 14).

El BM clasifica a los países según el porcentaje de alta tecnología que se encuentra en sus productos de exportación. Los productos de alta tecnología son considerados como los que incorporan un componente intensivo de I\&D, que incluye computadoras, productos farmacéuticos, instrumentos y equipos industriales, y productos de la industria aeroespacial, entre otros. En 2004, por ejemplo, el 34\% de las exportaciones 
de Irlanda fueron productos de alta tecnología; en Corea del Sur 33\%; en los Estados Unidos 32\%; pero en América Latina, Chile exportó sólo el 5\%; Brasil 12\%; y México 21\% (Banco Mundial, 2006) ${ }^{1}$. El análisis del Banco Mundial sugiere que la I\&D tiene un papel esencial en el desarrollo y que los países en desarrollo deben hacer una fuerte inversión en I\&D priorizando la innovación y mejorando la competitividad internacional. La Organización para la Cooperación y el Desarrollo Económico (OCDE) entiende que a fin de sostener el crecimiento económico es necesario una inversión en conocimiento (OCDE, 1998). Algunas voces intelectuales propusieron explícitamente que la NT puede ser estratégica para que los países menos desarrollados puedan saltar hacia el desarrollo. Un documento del Grupo de Trabajo sobre Ciencia, Tecnología e Innovación del Proyecto del Milenio de la Organización de Naciones Unidas hizo hincapié en la capacidad de la NT para la mejora de las condiciones de vida de los pobres (Juma y Yee-Cheong, 2005).

Dos tipos de políticas fueron necesarias para que este paradigma funcionara: un mecanismo para reconocer y tratar el conocimiento como un bien comerciable; y políticas para invertir en I\&D como una estrategia económica. La primera se llevó a cabo por la Organización Mundial del Comercio, que en 1994 estableció el Acuerdo sobre los Aspectos de los Derechos de Propiedad Intelectual (ADPIC), donde las patentes se convirtieron en garantía en las operaciones de comercio mundial. La segunda requirió inyecciones financieras específicas en los sectores educativo y científico. Durante la segunda mitad de la década de 1990 el Banco Mundial, la Organización de las Naciones Unidas (ONU), la Organización Mundial de Comercio, la OCDE y otras instituciones internacionales

1 En el caso de México, la presencia de producción de las maquiladoras y el fuerte comercio intra-firma entre empresas en México y los Estados Unidos sugiere la necesidad de un análisis cuidadoso (Delgado Wise e Invernizzi, 2002). 
impulsaron el paradigma de la economía del conocimiento como estrategia para el desarrollo, insistiendo en que los países en desarrollo deben invertir al menos el 1\% de su Producto Interno Bruto en I\&D. En América Latina, sólo Brasil llegó a esa tasa.

Desde finales de la década de 1990, el BM y otras instituciones han previsto la creación de una red global de Iniciativas Científicas del Milenio (ICM). Fueron concebidas como centros de excelencia en los países en desarrollo con el propósito de promover la investigación en C\&T con el mismo nivel de la infraestructura y recursos que existen en los países desarrollados (Macilwain, 1998). Los objetivos de la ICM fueron:

... para fomentar el crecimiento de las capacidades de investigación científica, empleando y estimulando al mejor talento en el país, como un factor clave para el desarrollo socio-económico. El programa prevé que la creación de Centros de Excelencia Científica que dará lugar al crecimiento de los Institutos Científicos y Núcleos Científicos bajo un proceso competitivo y transparente. Estos centros perseguirán la investigación científica de frontera, la formación de científicos y el establecimiento de vínculos con el sector productivo y otros acuerdos institucionales (ICM, s/d).

El proyecto de Chile fue el prototipo. En 1999 el BM destinó un préstamo de 5 millones de dólares para el primer período de dos años y medio, que fue acompañado por 10 millones de dólares del presupuesto nacional de Chile (ICM, s/d). Varios institutos de investigación en NT fueron creados mediante esta iniciativa a partir de 1999 y durante los años siguientes (e.g. Universidad Técnica Federico Santa María, Universidad Andrés Bello). Además de Chile, Brasil, México y Venezuela recibieron ICMs. En Brasil, cuatro Institutos del Milenio en diversas áreas de la NT fueron creados en 2001 (Instituto de Nanociencias, Instituto de Materiales Complejos, Red de Investigación en Sistemas de Chips, Microsistemas y Nanoelectrónica, y el Instituto Multidisciplinario de Materiales Poliméri- 
Cos) (ABDI, 2010); y en México, otro proyecto de NT fue fundado en San Luis Potosí (IPICyT) (Rushton, et al, 2009; Foladori e Fuentes, 2008). Estos centros de excelencia tuvieron que sobrevivir en países donde se destina menos del 0,5\% del Producto Interno Bruto a la C\&T, como son los casos de México y Chile, o algo mayor, como en Brasil (1\%) pero, en cualquier caso, estos porcentajes son bajos en comparación con los designados para C\&T en los países desarrollados.

La Organización de Estados Americanos también promovió el paradigma de la economía del conocimiento. La Cuarta Reunión Ordinaria de la Comisión Interamericana de Ciencia y Tecnología (COMCYT) de la OEA, celebrada en Washington DC en 2004, tuvo en cuenta el tema tratado en cuatro talleres previos realizados en diferentes países durante 2003-2004. El celebrado en Ecuador en 2003 (OST, 2004) abordó las políticas hemisféricas para el desarrollo científico y tecnológico en las Américas y destacó cuatro áreas estratégicas para la región: biotecnología, tecnologías limpias y energías renovables, redes de información y tecnología, y los materiales y la NT.

Otros ejemplos de organizaciones internacionales que fomentan la NT en el paradigma de la economía del conocimiento se pueden encontrar en la manifestación de varias reuniones celebradas en los últimos años. El Centro Internacional para la Ciencia y el Desarrollo Industrial de las Naciones Unidas organizó, en 2005, la conferencia “Diálogo NorteSur sobre Nanotecnología: Retos y Oportunidades", que se centró en promover la participación de los países en desarrollo en la NT (Brahic, 2005a, 2005b; Brahic \& Dickson, 2005). De particular interés fue el mensaje del presidente de la Academia de Ciencias del Tercer Mundo, Mohamed Hassan (2005), quien propuso la creación de Centros de Excelencia en África, señalando que el desarrollo de la C\&T es una necesidad para el éxito de los países en desarrollo. La misma opinión fue discutida por los lí- 
deres de los países industrializados (Grupo de los Ocho) a partir de 2000. Durante su reunión anual en Escocia en 2005, apoyaron la idea de la formación Centros de Excelencia en África para instigar la transferencia y el intercambio de C\&T entre países desarrollados y en desarrollo (Dickson, 2005). Más recientemente, en el taller realizado en Argentina en noviembre de 2010, la Unión de los países de Sudamérica (UNASUR) acordó una ruta para la Ciencia, Tecnología e Innovación, que incluye a la NT como un área estratégica (Hirschfeld, 2010).

El camino hacia una economía del conocimiento promovida por el BM, la OCDE y otras instituciones tiene, al menos, dos aspectos discutibles en cuanto a su potencial de desarrollo. La primera es que el escenario para desarrollar economías de base tecno-científica es a través de la competencia internacional en una economía abierta. La segunda es que la transición a una economía del conocimiento se puede lograr mediante nichos de excelencia, sin tener en cuenta el estatus educacional general de la población, la capacitación laboral y una política de distribución de los resultados económicos dentro de la clase obrera. Estas son proposiciones discutibles. Por un lado porque contradicen abiertamente la historia de la industrialización en América Latina desde la década de 1930 y hasta la década de 1970, que fue el único período histórico en el que tuvieron lugar el desarrollo y una industrialización relativamente sólida. Ese proceso de industrialización se basó en las teorías (dependencia económica) y prácticas que protegían el mercado interno, tendían a universalizar el acceso a la educación pública primaria, secundaria, técnica y universitaria, y apoyaban el proceso con fuertes inversiones estatales en infraestructura productiva con políticas de bienestar sin precedentes. Por otro lado, también contradicen la experiencia de desarrollo e industrialización-tardía de los llamados Tigres Asiáticos durante la década de 1980 - un ejemplo utilizado por muchos para ilustrar que es posible el desarrollo en los paí- 
ses en vías de desarrollo. Estas experiencias ocurrieron bajo fuertes estados proteccionistas que, contrariamente a lo que comúnmente se dice, abrieron sus economías gradualmente y en etapas, sólo después de que la competitividad se había logrado, en lugar de las políticas de choque y rápida apertura de mercado que la mayoría de los países de América Latina persiguieron en los 80s y 90s. Aquellos países también promulgaron una profunda reforma educativa para elevar el acceso y la calidad de los niveles básicos de enseñanza, y financiaron inversiones masivas externas y locales en infraestructura, mientras se supervisaron los compromisos con el sector empresarial, sancionándolos cuando fallaban².

\section{Iniciativas de nanotecnología en América Latina: en busca de la competitividad}

Las NT estallaron globalmente y en América Latina en una economía mundial caracterizada por mercados abiertos. Una economía abierta es aquella en la que se reduce la protección arancelaria a un mínimo o no existe, el Estado reduce los subsidios a la producción nacional, son abolidas las cuotas y permisos de exportación / importación, y la conversión de la moneda está mucho más vinculada a las fluctuaciones del mercado. La economía convencional considera que la competencia internacional desencadena un proceso de adquisición de las mejores prácticas y capacidades tecnológicas, por lo que las empresas orientadas hacia el mercado externo tienden a ser más eficientes que sus homólogas focalizadas en el mercado interior. El proceso es doble: la competencia con productores de primera línea fuerza la modernización de las tecnologías y procesos;

2 Para un análisis comparativo de las distintas interpretaciones del "milagro asiático de desarrollo" ver Burkett e Hart-Landsberg, 2003. 
al tiempo que los compradores internacionales, acostumbrados a productos de mejor calidad, establecen requisitos que ayudan a mejorar los productos de los vendedores y las condiciones de producción. Al final la competencia internacional siempre impulsa una cultura de innovación. Siguiendo este enfoque, la alta tecnología debe demostrar su valía a través de la competencia en un mercado abierto.

De los tres países analizados, México fue el primero en empezar a abrir su economía. A partir de 1984, el gobierno eliminó los permisos de importación para la mayoría de los bienes, redujo posteriormente la protección arancelaria y canceló el sistema de precios fijos para productos seleccionados. A finales de la década los aranceles proteccionistas se habían reducido de alrededor del 24\% en 1985 a 12\% en 1993. La posterior incorporación de México al Tratado de Libre Comercio de Norteamérica a mediados de los noventa abrió aún más la economía. En Argentina el proceso fue aún más rápido. Se inició en 1989 con la eliminación de los permisos y la reducción de los aranceles proteccionistas. En 1991 a excepción de los automóviles y productos electrónicos, la mayoría de las tarifas de Argentina no superaban el 20\%. De 1989 a 1993 las tarifas internacionales promedio disminuyeron del 39 al 15\%. La economía brasileña, históricamente cerrada, se abrió de pronto a la competencia externa en 1990. De 1990 a 1993 las tarifas internacionales promedio disminuyeron de 32 a 14\%, aunque en este caso los aranceles proteccionistas se redujeron de manera diferente, teniendo siete niveles, desde 0 a 40\%, y los bienes en el sector de tecnologías de la información permanecieron con altos aranceles durante varios años (Agosin y Ffrench-Davis, 1994). Tras la apertura del mercado, la inversión extranjera directa (IED) aumentó. En Argentina, la IED aumentó del 7\% del PIB en 1980 a 12\% en 1997. En Brasil, la IED pasó de 7\% al 16\% y en México del $4 \%$ al $13 \%$ en el mismo período. 
Durante la primera década del siglo 21, y después de una ola internacional, muchos países latinoamericanos incluyeron a la NT como un área estratégica para fomentar la competitividad. Este ha sido el caso no sólo de los países más grandes y más desarrollados como Brasil, México y Argentina, sino también de los pequeños países de América Central, y otros como Uruguay y Ecuador, y países medianos como Colombia, Perú y Venezuela.

El Ministerio de Ciencia y Tecnología (MCT) brasileño (que cambió de nombre a partir de agosto de 2011 para Ministerio de Ciencia, Tecnología e Innovación - MCTI) se movió rápidamente para establecer las bases para una política nacional de NT. A finales de 2000, el MCT y el CNPq (Consejo Nacional de Desarrollo Científico y Tecnológico) reunieron investigadores en el área para formar un taller y comenzaron a establecer una agenda para impulsar la NT (GT Nanotecnologia, 2003). La primera acción fue la financiación de cuatro Redes Cooperativas Multidisciplinarias de Investigación Básica y Aplicada sobre Nanociencias y Nanotecnologías, que conectó a más de 300 investigadores, 600 estudiantes de posgrado, 77 universidades y centros de investigación y 13 empresas, con el propósito de crear y consolidar la capacidad nacional en el campo (Toma, 2005). En 2004, un Programa para el Desarrollo de la Nanociencia y la Nanotecnología se incorporó al Plan Plurianual del MCT para el período 2004-2007. Dicho programa recomendó acciones para construir y apoyar los laboratorios y redes de investigación. Varios laboratorios fueron re-equipados y se crearon otros nuevos. Una serie de proyectos para la incubación de empresas de NT y las actividades de I\&D de empresas e instituciones de investigación han sido financiados por FINEP (Agencia de Financiamiento para Investigaciones y Proyectos - una entidad del MCT) desde 2004, y los Fondos Sectoriales - una fuente importante de fondos de I\&D dedicados al sector productivo- crearon una iniciativa transversal para la NT. El CNPq también apoyó el financiamiento de otras investiga- 
ciones en adición a las redes, en particular el programa para los doctores jóvenes, y su programa general de becas para maestría y doctorado. Diez nuevas redes de investigación cooperativa se financiaron desde 2005 hasta 2009 y 17 fueron lanzadas en 2010. A finales de 2008, el CNPq financió una nueva estructura institucional, el Instituto Nacional de Ciencia y Tecnología, destinado a ocupar una posición estratégica dentro del Sistema Nacional de Ciencia, Tecnología e Innovación. Los institutos se caracterizan por un formato de red de investigación que incluye varias instituciones de diferentes regiones del país, dirigidas por una institución de excelencia. Al menos 21 Institutos Nacionales de Ciencia y Tecnología de 123, están involucrados en la NT. El presupuesto asignado a la NT por el MCT entre los años 2004, cuando el programa de NT fue lanzado, y 2009 ascendió a 270 millones de reales (MCT 2008, Embrapa, 2009)33.

En México, el Programa Especial de Ciencia y Tecnología 2001-2006 (PECyT), que forma parte del Plan Nacional de Desarrollo 2001-2006, mencionó, por primera vez en documentos oficiales a la NT como un área estratégica en el desarrollo de los materiales avanzados. El PECyT destacó la necesidad de un Programa Nacional de Nanotecnología y una Red de intercambio científico en esta área (CONACYT, 2002)-el primero aún no se ha creado. En 2008, el Programa Especial de Ciencia, Tecnología e Innovación 2008-2012 también estableció a la NT como una de las nueve áreas prioritarias para el desarrollo de la C\&T. Según algunas estimaciones, entre 1998 y 2004, 14,4 millones de dólares en fondos públicos fueron para I\&D de NT (CIMAV, 2008). Para el año 2010, la implementación de una política de NT en México tomó forma en cuatro desarrollos importantes: la Red de Nanociencia y Nanotecnología (2009), la construcción de dos Laboratorios Nacionales de NT (2007), y el de-

3 La relación entre la moneda nacional (real) y el dólar fluctuó mucho durante esos años (en términos generales: 2,9 reales por dólar en 2004, 2.2 en 2006, 2.0 en 2007, 1.7 en 2008, y 1.8 en 2009). 
sarrollo de parques de C\&T donde estuvieran integrados las empresas, el gobierno y la academia. En el Estado de Nuevo León se ha creado un clúster de nanotecnología, con una incubadora de NT orientado hacia las necesidades de las maquiladoras. Para 2010, había más de 60 universidades o centros públicos de investigación en México con programas de I\&D en NT, y aproximadamente 500 investigadores involucrados.

En Argentina, la Secretaría de Ciencia y Tecnología estableció a la NT como uno de los ámbitos prioritarios de financiamiento público en 2003. El primer taller en NT se llevó a cabo en 2004, y en el mismo año la Agencia Nacional de Promoción Científica y Tecnológica (ANPCyT) hizo un llamado para la presentación de proyectos, incluyendo a la NT entre las áreas a ser financiadas. De los poco más de un millón de dólares asignados, 23\% fueron para proyectos de NT (García et al., 2011). En 2005, la Fundación Argentina de Nanotecnología (FAN) fue lanzada con un presupuesto federal de 10 millones de dólares para los siguientes 5 años. En 2006, el Gobierno anunció la apertura de la primera licitación para la financiación de iniciativas sobre NT en el marco del programa FAN. Para el año 2010, la Agencia Nacional de Promoción Científica y Tecnológica, a través de su programa FONARSEC (Fondo Argentino Sectorial), comenzó una nueva línea de financiamiento en tres áreas de las NT: nanomateriales, nanointermediarios y nanosensores, con un importe de 10 millones de dólares, pero con el requisito de que las presentaciones fuesen conjuntas con empresas, y éstas contribuyeran con, al menos, un $20 \%$ del costo total del proyecto (FONARSEC, 2010). En Argentina existen cuatro redes de grupos de NT, con alrededor de 200 investigadores en los siguientes ámbitos: NT molecular, nanomateriales, MEMS / NEMS (Sistemas Micro-Electromecánicos y Sistemas Nano-Electromecánicos), y, bio-materiales. También hay un grupo de trabajo sobre nanomedicina, aparte de aquellas redes. 
Aumentar la competitividad es el objetivo explícito de las políticas de NT en los tres países. El Grupo de Trabajo brasileño para Nanociencias y Nanotecnología, que elaboró el primer programa de NT, se justifica a fin de:

... fomentar las actividades de investigación, desarrollo de nuevos productos y procesos y la transferencia de tecnología entre la universidad y la empresa con miras a la innovación tecnológica a fin de fomentar la competitividad en la industria nacional (MCT, 2004a, p.143).

En el Programa Especial de Ciencia y Tecnología 2008-2012 de México la importancia de la NT junto con otros campos se justifica de la siguiente manera:

El sector de C y T establece como fundamental el desarrollo en este campo de la educación de calidad y el fortalecimiento de la ciencia básica y aplicada, desarrollo tecnológico e innovación para ayudar a mejorar el nivel de vida en nuestra sociedad y lograr una mayor competitividad (CONACYT, 2008, p. 25).

El anuncio de la creación de la Red de Nanociencia y Nanotecnología en el año 2009 dice lo siguiente:

La investigación en nanotecnología puede permitir a nuestro país la innovación necesaria para crear empresas de alta tecnología para aumentar nuestro nivel de competitividad y crear empleos bien remunerados (CONACYT, s / f).

La misma lógica se encuentra en el decreto que creó la Fundación Argentina de Nanotecnología en el año 2005:

... un programa para estimular, sentar las bases y promover el desarrollo de la infraestructura humana y técnica en Argentina para que, a través de sus propias actividades relacionadas, pueden establecer las bases que permitan al país para competir a nivel internacional en la aplicación y desarrollo de las micro y nanotecnologías que aumenten el valor obtenido de los productos para consumo interno y de exportación (Decreto Presidencial 380/2005). 
Compromisos similares al uso de la NT para aumentar la competitividad internacional se pueden encontrar en los programas de C\&T de otros países de América Latina. La política científica y tecnológica global se basa en la idea de que la competitividad es el instrumento clave para el desarrollo.

\section{Creando condiciones para la investigación de excelencia en nanotecnología}

Esfuerzos significativos se realizaron en los tres países hacia la mejora de equipamiento técnico para cumplir con las condiciones de investigación más competitiva. Aunque no existe una base de datos que recopile la adquisición de nuevos equipos en los laboratorios de NT (ni siquiera dentro de cada país) la tabla I (Anexo) es un esfuerzo por ilustrar lo que existe en los tres países. El propósito de esto no es comparar a los laboratorios o los países en términos de cantidad o calidad. Puesto que la NT es una plataforma tecnológica que cruza las diferentes áreas, algunos instrumentos son de amplio uso a través de ellas. Otros equipos, sin embargo, son más específicos para cada área. El equipo necesario para un laboratorio bio-médico es diferente en calidad, cantidad y valor de un laboratorio de ciencias de los materiales, un laboratorio de electrónica, y así sucesivamente. Además de las diferencias técnicas y de financiamiento, el instrumental no necesariamente demuestra la importancia del grupo de investigación en términos de indicadores de producción en C\&T, ya que algunos grupos son más académicos y orientados hacia la investigación básica, otros a cumplir los requisitos industriales, otros son instalaciones de servicio y la cantidad de investigadores varía entre ellos. Las redes y asociaciones entre instituciones también complican el panorama, ya que grupos de investigación sin instalación de laboratorio pero con acceso a instalaciones de otros centros se puede desarrollar sin infraestructura y equipamiento propios. En la cuanti- 
ficación de esta imagen, nuestra metodología contempla estas dificultades introduciendo un enfoque de cronograma de modernización, para resaltar la tendencia de las inversiones en cada país, aunque los datos temporales no estuvieron disponibles en todos los casos. Una dificultad adicional es el hecho de que muchas de estas instituciones se han actualizado en términos de equipo en los últimos años y, en algunos casos, a los laboratorios actualizados se les dio nuevos nombres. La Tabla I registra la fecha de inauguración del laboratorio cuando se trata de uno nuevo, pero la fecha de la actualización cuando se trata de un uno pre-existente.

Brasil tiene la única instalación de Luz Sincrotrón (LNLS) en América Latina, con sede en Campinas, Estado de São Paulo, en uso desde 1997. La construcción de un segundo Laboratorio de Luz Sincrotrón (SIRIUS) se inició en 2010 en un área vecina, pero en este caso con tecnología nacional y en colaboración con Argentina. La luz sincrotón, producida por la conversión en luz de partículas artificialmente aceleradas, permite explorar la estructura de la materia. Es ampliamente utilizado para el estudio de la estructura atómica de los líquidos y sólidos, de las reacciones químicas y de estructuras biológicas y moleculares. LNLS es una instalación multiusuario con un alcance internacional. Dentro del área del LNLS, el Centro Nacional de Nanociencias y Nanotecnologías César Lattes fue creada en 2008, con laboratorios de microscopía avanzada. Por el tamaño y la cantidad de usuarios (alrededor de 1500 investigadores por año) el LNLS no tiene parangón en América Latina. Otro laboratorio de NT es el del Centro Nacional de Nanometrología, creado en 2005 como parte del INMETRO (Instituto de Metrología) en Río de Janeiro. Para 2008, se equipó con alta tecnología y equipos muy caros, como el microscopio electrónico de barrido de transmisión Titán, capaz de ofrecer imágenes de resolución atómica sub-Angstrom $\left(10^{-10} \mathrm{~m}\right.$ ). El INPE (Instituto Nacional de Investigaciones Espaciales) cuenta con un laboratorio de NT (LAS - Labo- 
ratorio Asociado de Sensores y Materiales) que también se ha actualizado en 2007. El Centro Brasileño de Investigaciones Físicas (CBPF) inauguró su laboratorio de Nanoscopía en 1994, pero solo inauguró el LabNano una enorme instalación multiusuario en Río de Janeiro - en el año 2005. Otro laboratorio público de NT es el CenPRA (Centro de Investigación Renato Archer) del MCT, que se especializa en micro / nano electrónica. En la misma área de investigación, en 2008, fue creada Ceitec SA (Centro Nacional de Tecnología Electrónica Avanzada) en Rio Grande do Sul, una planta de producción de semiconductores y chips con el objetivo de abastecer el MERCOSUR. Petrobrás, la empresa petrolera estatal tiene laboratorios de alta tecnología para la NT, y en 2006 creó una Red de 10 instituciones de investigación que trabajan juntas en NT en áreas de investigación de petróleo. En 2009, un laboratorio del Ministerio de Agricultura, el LNNA (Laboratorio de Nanotecnología y Nanociencias para Agronegócios) fue inaugurado por EMBRAPA, la Corporación Brasileña de Investigación Agropecuaria, en el estado de São Paulo. Otro laboratorio, que también trabaja en NT como una instalación multiusuario para todo el país es el LNCC (Laboratorio Nacional de Computación Científica), ubicado en Río de Janeiro. Específicamente orientado a la industrialización de la región noreste está el CETENE (Centro de Tecnologías Estratégicas del Nordeste), creado en 2005 en Pernambuco. En un ámbito más regional y local hay más de 20 universidades, principalmente estatales y federales, así como centros de investigación pública en NT, muchos de ellos con laboratorios bien equipados; tales como la UFRGS (Universidad Federal de Rio Grande do Sul) en Porto Alegre que tiene al menos siete laboratorios que trabajan en NT. A partir de 2008, el Ministerio de Ciencia y Tecnología organiza la investigación científica brasileña en redes llamados Institutos Nacionales de Ciencia y Tecnología; estos institutos permiten 
a los investigadores trasladarse a centros con infraestructura y establecer iniciativas conjuntas de investigación.

México tiene más de 50 universidades y centros públicos de investigación de NT. Entre los más relevantes está la Universidad Nacional Autónoma de México (UNAM), que cuenta con más de una docena de departamentos e institutos en la Ciudad de México y otras universidades del país donde se está haciendo NT. En el campus de la Ciudad de MéxiCo, el Instituto de Física tiene, desde 2002, el Laboratorio de Microscopía Central. Cerca de la frontera del Pacífico con los Estados Unidos en Baja California Norte, el campus de la UNAM en Ensenada creó el CN y N (Centro de Nanociencias y Nanotecnología) en 2008, con un laboratorio bien equipado; y el campus de Querétaro tiene el CFATA (Centro de Física Aplicada y Avanzada tecnología), que ha estado investigando NT desde 1996. Otros departamentos de la UNAM, tales como Ciencias de los Materiales, Química, Astrofísica, también tienen centros de investigación. Sumando todos los investigadores en NT en el país, la UNAM cuenta con aproximadamente una quinta parte de ellos. Otra gran universidad pública es la UAM (Universidad Autónoma Metropolitana) en el Área Metropolitana del Distrito Federal. Dos de los tres campus cuentan con laboratorios en NT. El de Iztapalapa (UAM-I) es la sede del Laboratorio de Resonancia Magnética Nuclear desde 1995, actualizado significativamente en 2006 y 2009; el Laboratorio de Nanotecnología e Ingeniería Molecular (desde 2004), un laboratorio de nanotecnología química, y, desde 2007, el CI3M (Centro Nacional de Investigación en Imagenología e Instrumentación Biomédica). El campus de Xochimilco (UAM-X), en colaboración con la Secretaría de Salud construyó el Laboratorio de Nanotecnología aplicada a la Medicina (2004-INNN "MVS"). Un tercer gran centro público de investigación, de alcance nacional, es el Instituto Politécnico Nacional (IPN). Su centro de investigación CINVESTAV cons- 
truyó, en 2005, el LANGEBIO (Laboratorio Nacional en Genómica para la Biodiversidad) en el campus de Guanajuato, y en 2009, el Centro de Nanociencias de Micro y Nanotecnología en el campus de Querétaro. Otros campus del IPN cuentan con laboratorios especializados, como el de semiconductores en Guadalajara (CTS-Centro de Tecnología de Semiconductores) y en Ingeniería Electrónica en Zacatenco, Ciudad de México. Tres microscopios Titán fueron adquiridos por diferentes centros de investigación mexicanos. El primero en 2006, por el Instituto Mexicano del Petróleo (IMP) para su Laboratorio de Microscopía de Ultra Alta Resolución Electrónica en la Ciudad de México. Los otros dos en 2008: uno por el CIQA para su LabMic, uno de los varios centros de investigación del CONACYT, especializado en Química Aplicada y ubicado en Saltillo, Coahuila; y el otro por la UANL (Universidad Autónoma de Nuevo León) en Monterrey, en el CIIDIT (Centro de Innovación de Investigación y Desarrollo en Ingeniería y Tecnología), en un Parque Científico y Tecnológico que es también la sede del Cluster de Nanotecnología. Otros dos Centros de Investigación CONACYT ganaron una convocatoria para laboratorios nacionales en 2006, por lo que el CIMAV en Chihuahua creó el NaNoTe$\mathrm{CH}$ (Laboratorio Nacional de Nanotecnología), mientras que el IPICyT en San Luis Potosí creó el LINAN (Laboratorio Nacional de Investigación en Nanociencia y Nanotecnología); ambos destinados a cubrir las demandas nacionales. Sin embargo, otros centros públicos cuentan con laboratorios importantes, como el del Instituto Nacional de Astrofísica, Óptica y Electrónica (INAOE) en Puebla, que abrió sus puertas en 2001 y ha ido actualizando su laboratorio en los últimos años hasta el recientemente creado Laboratorio para la Innovación en Nanotecnología y MEMS (2010).

Argentina está muy por detrás de los dos países anteriores en términos de equipamiento técnico y construcción de infraestructura para la NT. Sin embargo, algunos laboratorios ya existentes se han actualizado duran- 
te la década, y se han creado algunos otros nuevos. Un Centro Virtual de Nanociencias y Nanotecnología fue creado en 2005, con el fin de coordinar los esfuerzos que incluyen el Centro Nacional de Energía Atómica, la Universidad de Buenos Aires y la Universidad de La Plata / INIFTA. Las tres instituciones principales que investigan NT son: la Comisión Nacional de Energía Atómica (CNEA) en sus campus en Bariloche y Constituyentes, la Universidad de Buenos Aires (UBA) y la Universidad Nacional de La Plata (INIFTA -el Laboratorio de Microscopía y Superficies Físico-Químicas es un centro de investigación abierto en 1992 por el Conicet / UNLP). INQUIMAE (Instituto de Química y Física de Materiales, Medio Ambiente y Energía) es un centro de investigación del Conicet / UBA que inició en 1995 en Buenos Aires. El Instituto de Nanociencia y Nanotecnología, creado en 2007 por el Centro Atómico Bariloche, fue actualizado en 2009. Otros laboratorios de NT incluyen el Centro para la Ciencia y Tecnología de los Materiales, de nueva creación; además de un proyecto conjunto del CONICET con la Universidad Nacional de Mar del Plata. Otros laboratorios son el especializado en medicina (Diseño Estratégico para la Focalización de Medicamentos) con sede en la Universidad Nacional de Quilmes (UNQ); el Laboratorio de Ingeniería de Tejidos, Medicina Regenerativa y Terapias Celulares, por el Gobierno de la Provincia de Buenos Aires; un laboratorio del ministerio de Defensa, y el del Instituto Nacional de Tecnología Industrial, con su laboratorio actualizado en 2005.

La decisión de aumentar las instalaciones de alta tecnología y equipo está acompañada por varios de los nuevos programas de postgrado en NT. En México, por ejemplo, para el año 2010 se llevaron a cabo cerca de 33 programas de maestría y doctorado en NT, o con especialización en NT, y alrededor de 7 cursos de licenciatura. Nada en cantidad similar ocurrió en Brasil, donde sólo un par de cursos de postgrado se pusieron en marcha (e.g. Universidad Federal de ABC, Sao Paulo y UNIFRA, RGS), 
y otros varios cursos de postgrado tienen líneas de investigación en NT (e.g. PUC / Río de Janeiro); y fueron creados otros tres cursos de licenciatura en NT en 2010 y 2011. Argentina es similar a Brasil, donde sólo fueron creadas unas pocas especializaciones de post-grado en NT. Pero la diferencia de los dos últimos países con México probablemente se debe más a los diversos procedimientos de gestión institucional para crear nuevos cursos que a la falta de voluntad de avanzar en esa dirección.

Los esfuerzos para crear o mejorar las instalaciones de investigación con miras a la investigación de clase mundial, como un elemento principal de las políticas de NT dirigidas a incrementar las capacidades competitivas de los países, necesitan ser puestos en el contexto de los cambios significativos que experimentó la organización del trabajo científico desde los años noventa y que tenía particulares implicaciones para los investigadores de estos países en desarrollo. Un elemento determinante fue la Internet. Las NT son la primera revolución tecnológica que se da en el marco de un amplio y accesible uso de la Internet por parte de los investigadores. Las principales universidades de los tres países comienzan a tener acceso más amplio a la Internet en sus locales de investigación durante la década de noventa. Una segunda condición fue el acceso más amplio a revistas científicas internacionales, a través del contrato de servicios de revistas científicas electrónicas lo que amplió radicalmente las condiciones de acceso a las principales publicaciones científicas mundiales. Surgen también portales de revistas electrónicas en español y portugués, siendo Scielo el más importante. Con esto se dan las bases materiales para que los investigadores de Latinoamérica puedan conectarse mucho más activamente con sus pares en otros países del mundo sin tener que moverse de su lugar de trabajo.

Esta facilidad material para globalizar el conocimiento se suma a las políticas de C\&T que se venían implementando, relativas a evaluar a los investigadores por su productividad, medida según los criterios de los 
países desarrollados. Se jerarquizan las revistas científicas según el impacto de citación, y se pondera a los investigadores por la productividad en dichas revistas. El resultado es que los investigadores deben de ajustarse al lenguaje (publicar en inglés) y a los temas que las revistas internacionales destacan. La Capes y el CNPq en Brasil, el Conicet en Argentina, y el CONACYT en México, que son las instituciones rectoras de las políticas de C\&T van encauzando a la investigación científica en los padrones internacionales, lo cual significa no sólo padronizar las formas y exigencias de la investigación, sino también los temas e intereses.

\section{Incorporando al sector privado}

Una característica bien conocida de las empresas privadas en América Latina es su falta de innovación, que representa un desafío para el paradigma de la innovación basado en la empresa privada como el motor del proceso. Es bien sabido que después de la investigación y desarrollo de prototipos, pero antes de la producción en masa y la comercialización, existe lo que se llama vulgarmente el valle de la muerte. De hecho, como casi toda la I\&D de América Latina está financiada públicamente, la brecha es aún mayor, dado que casi no hay experiencia en investigación científica aplicada, ni la tradición cultural de científicos que se vuelvan ejecutivos. Para superar este problema se han implementado varios incentivos económicos para asociar firmas privadas con la I\&D pública.

En Brasil, la nueva política de C\&T incrementó el gasto público en más del doble entre 2000 y 2006, para llegar a cerca de 7 mil millones dólares, de los cuales la mayor parte se destinó a actividades de innovación en empresas. Por ejemplo, el CNCPq incrementó significativamente el número de becas y los fondos para investigación en proyectos de desarrollo tecnológico de colaboración empresa-universidad. Por otro lado, 
los programas del FINEP para apoyar las actividades innovadoras de las empresas incluían varios mecanismos como subsidios no reembolsables, incentivos fiscales, fondos reembolsables, y fondos compartidos (cerca de un tercio de la investigación de esta agencia ha tenido contraparte de financiamiento de las empresas). El Ilamado de propuestas de investigación entre empresas e instituciones de investigación y las subvenciones económicas fueron los principales instrumentos utilizados para promover la investigación conjunta en NT entre universidades y empresas.

En México, los programas públicos de financiamiento fueron reorientados hacia colaboraciones con el sector privado, el cual pasó de percibir el 10\% del financiamiento público en I\&D en 2002 a $21 \%$ en 2006. A partir de 2008, con el anuncio del Programa Especial de Ciencia y Tecnología 2008-2012, la exigencia de empresas junto a centros públicos de I\&D pública se volvió más explícita, y fueron creados varios nuevos programas de financiamiento para empresas pequeñas, medianas y también grandes corporaciones. La Ley de Ciencia y Tecnología, modificada en 2009, estableció que los centros de investigación públicos pueden y deben fomentar spin-offs privados. Esto permite también a los investigadores mantener hasta el $70 \%$ de las regalías por derechos de propiedad intelectual, alentando a los investigadores a convertirse en empresarios.

En Argentina, un cambio hacia el financiamiento de la I\&D junto al sector empresarial o directamente a las empresas privadas está presente desde finales de la década de 19904. El instrumento de financiamiento más reciente, el Fondo Argentino Sectorial (FONARSEC), lanzado en 2010 con fondos del Banco Interamericano de Reconstrucción y Desar-

4 La integración de las empresas transnacionales con la I\&D latinoamericana en NT probablemente se inició en 2000 con un viaje de financiación por parte de Motorola. La compañía financió proyectos en Brasil (Sao Paulo), en Bariloche (CNEA) (Argentina) y en México (INAOE, Puebla). 
rollo, inició una financiación específica para la investigación en nanomateriales, productos intermedios y nano-nanosensores (FS Nano) pero cuyos proyectos deben ser presentados junto a empresas.

Aunque la mayoría de los instrumentos de política están orientados a la incorporación de la empresa privada, el Estado en los tres países todavía tiene una presencia dominante en algunos sectores clave y de importancia económica y social, como salud, energía, agua y transporte, en los que hay no sólo centros de investigación sino también instalaciones de producción. Esto ofrece la posibilidad de conectar la C\&T con la producción y el consumo sin pasar por el valle de la muerte que se produce entre los procesos de C\&T y las esferas de mercado, acentuado allí donde la investigación es pública y la producción privada. En los tres países existen centros de investigación públicos en áreas energéticas estratégicas con laboratorios de I\&D en NT (en Argentina la Comisión Nacional de Energía Atómica; en Brasil la petrolera estatal Petrobras; en México el Instituto Mexicano del Petróleo). Ejemplos similares pueden encontrarse en el área de salud (FIOCRUZ en Brasil, o el Instituto Nacional de Neurología y Neurocirugía de la Secretaría de Salud en México). Algunas otras investigaciones se realizan en áreas de protección y riesgos ambientales, pero este es más el interés personal de los grupos de investigación que una política pública explícita.

\section{Evaluación de las políticas de nanotecnología: reflexiones finales}

Cuando para inicios del siglo XXI la NT irrumpió en escena, el contexto económico de los países Latinoamericanos estaba caracterizado por la reorientación de la economía hacia un mercado internacional abierto que inició a finales de los ochenta. Visto en perspectiva histórica el desarrollo de América Latina tuvo un punto de inflexión en la década de 
los ochenta, conocida como la década perdida debido al crecimiento del endeudamiento externo que terminó con devaluaciones galopantes de las monedas e índices negativos de crecimiento relativo en la región. En el cono sur de América Latina esta década se caracterizó por represivas dictaduras militares. La crisis económica y las políticas represivas debilitaron las organizaciones sindicales y sociales y facilitaron la incorporación de los países de América Latina al GATT (General Agreement on Tariffs and Trade) y la liberalización de sus economías. Así se entró, durante los noventa, en un periodo de políticas económicas neoliberales, donde el libre mercado y la globalización financiera marcaban el camino. Para finales de la década del noventa Argentina, Brasil y México tenían economías significativamente más abiertas que diez años antes y habían pasado por un proceso de desindustrialización. La educación se volvió cada vez más privada y selectiva. Por más de un siglo, la educación había sido vista en Latinoamérica como un requisito para calificar a la clase obrera para el desarrollo industrial. Era una tarea del gobierno y vista por la sociedad civil como una condición para la ciudadanía y una clave para superar las diferencias étnicas y de clase. Desde la década de los 90, siguiendo el paradigma neoliberal, la educación se volvió un producto para ser adquirido en el mercado, y un rápido proceso de privatización comenzó a competir en todos los niveles educacionales con la educación pública.

Las políticas de C\&T también se ajustaron al proceso general de privatización. Se integró la investigación con financiamiento público a la producción privada; se orientó la investigación hacia mejorar la competitividad internacional; se crearon centros de excelencia con financiamiento público para apoyar la industria privada; y cambiaron los criterios de evaluación de las instituciones de investigación y de los propios investigadores y se modificaron los salarios. Se presionó por resultados cuantitativos medidos en artículos científicos y libros publicados, y por la publi- 
cación en revistas internacionales, lo que llevó a privilegiar los temas de investigación del mundo desarrollado, que no siempre coinciden con las necesidades nacionales de los países en desarrollo. Complementos salariales por productividad fueron otorgados en Argentina y México mediante los sistemas nacionales de investigadores, y en Brasil mediante las becas de productividad. Al mismo tiempo, la difusión de la Internet permitió la generalizaron el trabajo en redes internacionales, reforzando los vínculos locales con las agendas internacionales de investigación.

Cuando a principios del siglo XXI las NT irrumpen como una nueva revolución tecnológica, no lo hacen en un contexto económico orientado hacia el desarrollo nacional, sino en medio de una fuerte presencia de corporaciones transnacionales, privatización de empresas públicas y orientación hacia el mercado externo. El contexto también se caracterizaba por baja combatividad social, donde la presencia de sindicatos y movimientos sociales no tiene incidencia en las políticas científicas.

Sin organizaciones sociales de base local que participen en políticas de C\&T, y con una creciente presencia de empresas nacionales e internacionales asociándose con centros de investigación públicos, surge la duda razonable de la capacidad que tales estrategias de C\&T puedan tener para promover el desarrollo de la región contribuyendo a reducir la desigualdad y la pobreza. Incluso si los tres actores que participan en la estrategia de C\&T (Estado, empresarios y académicos) son exitosos en impulsar la innovación y la competitividad, esto no significa que la población en general se vea beneficiada. El proceso puede terminar ganando nichos de mercado, incluso competitividad, al tiempo que aumentando la desigualdad social. El énfasis que los gobiernos están poniendo en la integración de la I\&D pública con empresas privadas hace evidente que la lógica de estas políticas es lineal, al equiparar competitividad con crecimiento económico y desarrollo social. Las agendas de NT en América Latina han 
dejado de lado tres temas claves del desarrollo: la participación de sindicatos y movimientos sociales; la calificación de la fuerza de trabajo; y, la confianza del consumidor.

Sin una orientación política explícita hacia las demandas nacionales y locales los beneficiarios potenciales de la NT dependerán del goteo del crecimiento orientado a la exportación, que es una estrategia de desarrollo cuestionable. Para incorporar nuevos objetivos a la política de NT, así como para estimular el desarrollo de las áreas que tendrán un impacto más directo en el nivel de vida de la población se requiere la incorporación de otros grupos sociales en la toma de decisiones políticas, además del Estado, la academia y los empresarios. La presencia activa de los sindicatos y las ONGs pueden fortalecer y ampliar algunas de las iniciativas de investigación que ya están en curso en algunas instituciones, fundamentalmente en la salud. Nuevos temas se pueden incluir para responder a las demandas sociales. Algunos sindicatos y organizaciones sociales son conscientes de la evolución de la NT y han estado participando activamente en la difusión de sus preocupaciones y posiciones, pero sin canales de participación dentro de la estructura formal de la política científica (Foladori e Invernizzi, 2008; Foladori, Bejarano e Invernizzi, en prensa).

El segundo tema que debe ser incorporado a la agenda de de la NT es la calificación de la mano de obra. Con el fin de ser capaces de transferir la I\&D "de excelencia" a la producción es necesario un proceso permanente de capacitación de la mano de obra. Un pensamiento común es que la NT se encuentra todavía en las primeras etapas de la I\&D y que todavía hay tiempo suficiente para formar a los trabajadores una vez que comiencen los procesos de producción. Sin embargo en Brasil hay más de 150 empresas con I\&D o produciendo con NT, muchas de ellas ya comercializando productos (Invernizzi, 2011). En México la lista supera las 60 (Zayago y Foladori, 2010). También está el caso de varias empresas 
que están o podrían estar comprando nano materias primas en el mercado internacional para incorporar en los procesos de producción, lo que pone a los trabajadores en contacto con productos nano incluso antes de que la I\&D se realice a nivel local. También está la cuestión de los técnicos de los laboratorios que incorporan la NT, como en el área farmacéutica o biomédica. Estas personas no tienen los conocimientos, la infraestructura, los procedimientos de seguridad, ropa adecuada y otros factores para garantizar la correcta manipulación de los nanomateriales.

El tercer tema es el de la confianza de los consumidores en las nuevas tecnologías. En este sentido las implicaciones sociales y de riesgo de la NT son un área fundamental que debe abordarse. La falta de participación de las organizaciones sociales en el proceso de decisión de la C\&T, así como la indiferencia por parte de las agencias de C\&T en los problemas de riesgo pone el proceso de desarrollo de la NT distante de las necesidades sociales. Portavoces de la política e incluso científicos a menudo argumentan que no existe prueba definitiva con respecto a los nano-riesgos. Sin embargo, el NanoCeo, un banco de artículos científicos sobre los riesgos relacionados a la NT -que no es exhaustivo- ha reunido suficiente material que debe llevar a la preocupación y precaución al tratar con la NT. Una compilación de 1991 a noviembre de 2010 da el siguiente resultado: 176 artículos científicos relacionados con riesgos de los nanotubos de carbono, 216 de riesgos de las nanopartículas de plata, 81 de las nanopartículas de titanio, 72 de fullerenos y buckyballs, y 49 de puntos cuánticos, todos ellos nanopartículas o nanoestructuras de entre las más comunes en los productos del mercado. Otra institución que reúne información sobre los riesgos asociados con NT es el Consejo Internacional de Nanotecnología (ICON), que forma parte de la Universidad de Rice en Estados Unidos. De 2000 a 2010 su base de datos registró un aumento en los trabajos científicos sobre los riesgos para la salud humana 
y el medio ambiente de la NT, que pasó de alrededor de un centenar en 2005 a más de 550 en 2010. ${ }^{5}$ No obstante las claras evidencias de la necesidad de una política precautoria, el modelo de orientación hacia los negocios ha llevado a la escasa consideración de los potenciales riesgos.

En resumen las políticas de NT no han contemplado ni la participación de organizaciones sociales y sindicatos en la elaboración de políticas y de prioridades nacionales, ni tampoco la necesidad de integrar la calificación de la fuerza de trabajo como un aspecto del cambio tecnológico. Esto deja de lado como fuerza de apoyo activo a la clase obrera. Tampoco estas políticas han dado importancia a los potenciales riesgos a la salud y el medio ambiente de las NT, lo cual deja por fuera al consumidor como sector fundamental del desarrollo. Tímidamente algunos países de la región comienzan a considerar estos aspectos, pero como resultado del movimiento internacional y a cuentagotas.

\section{Referencias}

ABDI (Agencia Brasileira de Desenvolvimento Industrial). Panorama Nanotecno-

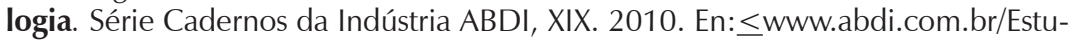
do/Panorama\%20de\%20Nanotecnologia.pdf > Visitada feb., 10, 2011.

AGOSIN, Manuel R.; FFRENCH-DAVIS, R. Liberalización comercial y desarrollo en América Latina. Nueva Sociedad, 133. 1994. p. 54-71.

BM (Banco Mundial). Reporte Mundial de Desarrollo. New York, Oxford: University Press, 1991.

BM (Banco Mundial). Indicadores Mundiales de Desarrollo 2006. CD-ROM. Washington, D.C., 2006.

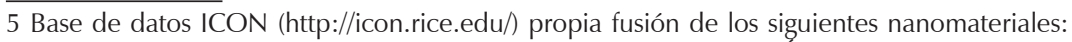
Carbono o Metal u Orgánicos / Polímeros o Semiconductores o de Óxido o Múltiple u Otras / No Especificadas] + Peligro para los siguientes grupos de los trabajadores [Industriales / Trabajadores de Investigación o Los consumidores o la Población en General o los Ecosistemas u Otro / No Especificado] + Artículos de Revistas por Revisar + Ingeniería. 
BM (Banco Mundial). Construyendo Economías del Conocimiento, Estrategias Avanzadas para el Desarrollo. Washington DC, El Banco Mundial. 2007. En:_<http:// siteresources.worldbank.org/KFDLP/Resources/461197-1199907090464/BuildingKEbook.pdf $>$. Visitada marzo 18, 2011.

BRAHIC, Catherine \& DICKSON, David. Helping the poor: the real challenge of nanotech. SciDev.Net February 21, 2005. En: < http://www.scidev.net/content/ editorials/eng/helping-the-poor-the-real-challenge-of-nanotech.cfm $>$. Visitada febrero,10, 2011.

BRAHIC, Catherine. Developing world 'needs nanotech network'. SciDev.Net Febrero 11, 2005. 2005a. En: <www.scidev.net/news/index.cfm?fuseaction=printar ticle\&itemid=1923\&language =1> Visitada febrero10, 2011.

BRAHIC, Catherine. Nanotech revolution needs business know-how. SciDev.Net Febrero 18, 2005. 2005b. En: < http://www.scidev.net/News/index.cfm?fuseactio $\mathrm{n}=$ readnews\&itemid =1938\&language $=1>$. Visitada febrero10, 2011.

BURKETT, Paul \& HART-LANDSBERG, Martin. A Critique of "Catch-Up" Theories of Development. Journal of Contemporary Asia, no 33, v. 2, p. 147-171. 2003.

CIMAV (Centro de Investigaciones en Materiales Avanzados). Diagnóstico y Prospectiva de la Nanotecnología en México. 2008. En: < http://www.nanotech.cimav.edu.mx/> Visitada enero 14, 2011.

CONACYT (Consejo Nacional de Ciencia y Tecnología). Programa Especial de Ciencia y Tecnología 2001-2006. Consejo nacional de Ciencia y Tecnología. [Véase también Vol. II]. 2002.

CONACYT (Consejo Nacional de Ciencia y Tecnología). Programa Especial de Ciencia, Tecnología e Innovación 2008-2012. México: CONACYT._2008. En: <http://www.siicyt.gob.mx/siicyt/docs/contenido/PECiTI.pdf $>$ Visitada oct. 31, 2009.

CONACYT (Consejo Nacional de Ciencia y Tecnología). Red Temática de Nanociencias y Nanotecnología. Dirección de Redes. DAIC. México: CONACYT. s/f. En: $\quad<$ http://www.conacyt.mx/Redes/Redes-Tematicas/Red-Nanociencias-y-Nanotecnologia.pdf $>$ Visitada febrero 10, 2010.

Decreto Presidencial 380/2005. República Argentina. Autorízase al Ministerio de Economía y Producción a constituir la Fundación Argentina de Nanotecnología. 2005. En: <http://www.fan.org.ar/acerca_estatuto.htm> Visitada oct., 31, 2009.

DELGADO WISE, R. \& INVERNIZZI, N. México y Corea del Sur: Claroscuros del crecimiento exportador en el contexto del globalismo neoliberal. Aportes, Revista Mexicana de Estudios sobre la Cuenca del Pacífico, II, 2, 4, pp. 63-86. 2002. 
DICKSON, David. Líderes del G8 dan impulse indirecto para la ciencia en África. SciDevNet, Septiembre 3. 2005. En: < http://www.scidev.net/news/index.cfm?fu seaction $=$ printarticle\&itemid $=2549 \&$ language $=1>$ Visitada febrero 10, 2011.

EMBRAPA. Coordenador do MCT anuncia recursos de R\$ 1 milhão para popularização da nanotecnologia, 14/09/2009. 2009. En: <http://www.embrapa.br/ imprensa/noticias/2009/setembro/3a-semana/coordenador-do-mct-anuncia-rercursos-de-r-1-milhao-para-popularizacao-da-nanotecnologia/ > Visitada febrero 20, 2011.

FOLADORI, Guillermo \& INVERNIZZI, Noela. The Workers Push to Democratize Nanotechnology. En E. Fisher, C. Selin and J. Wetmore, eds, The Yearbook of Nanotechnology in Society.UK: Springer, pp. 23-36. 2008.

FOLADORI, Guillermo; BEJARANO, Fernando \& INVERNIZZI, Noela. Trabajadores, consumidores, y el riesgo de las nanopartículas manufacturadas. La negociación multilateral del SAICM en América Latina y el Caribe. (en prensa).

FOLADORI, Guillermo \& FUENTES, Verónica. Nanotechnology in Chile. Towards a Knowledge Economy. En: Foladori, G. \& Invernizzi, N. (Eds.) Nanotechnologies in Latin America. Berlin: DietzBerlin, 2008. pp. 68-83.

FONARSEC. Bases de la convocatoria Fondo Sectorial de Nanotecnología FSNano-2010. Buenos Aires: ANPCyT, MINCyT. 2010.

GARCÍA, Marisa; LUGONES, Manuel \& REISING, Ailin María. Conformación y desarrollo del campo nanotecnocientífico argentino: una aproximación al estado de la cuestión desde el estudio de los instrumentos de promoción científica y tecnológica. En: Foladori, Záyago \& Invernizzi (comp.). Perspectivas del desarrollo de las nanotecnologías en América Latina. (en prensa).

GT Nanotecnologia (Grupo de Trabalho em nanociência e nanotecnologia). Ministério da Ciência e Tecnologia. Brasil. Nanotecnologia. Desenvolvimento da nanociência e da nanotecnologia. Proposta do Grupo de Trabalho criado pela Portaria MCT no 252 como subsídio ao Programa de Desenvolvimento da Nanociência e da Nanotecnologia do PPA 2004-2007. 2003. En: http://www.mct.gov. br/upd_blob/0002/2361.pdf Visitada oct., 31, 2009.

HASSAN, Mohamed. Nanotechnology: Small things and big changes in the developing world. Science, 309, 5731, pp.65-66. 2005.

HIRSCHFELD, Daniela. Latinoamérica define hoja de ruta para innovación. SciDevNet. Dic., 13, 2010. En: <http://www.scidev.net/en/science-and-innovation-policy/latin-america-sets-a-roadmap-for-innovation-.html> Visitada marzo 16, 2011.

ICM (Iniciativa Científica Milenio). Iniciativa Científica Milenio. Memoria Bianual 1999-2000. Santiago: MIDEPLAN. s/f a. En:<http://www.mideplan.cl/ milenio/?q=node/34 > Visitada mayo 23, 2007. 
INVERNIZZI, Noela. Nanotechnology in Brazilian firms: assessing potential implications for labor. Emerging Technologies/Emerging Economies. Nanotechnology for Equitable Global Development. Oxford: Routledge, (en prensa).

JUMA, Calestous \& YEE-CHEONG, Lee (coord.). Innovation: applying knowledge in development. London, Sterling, Va.: Earthscan, Millennium Project._2005. En: www.unmillenniumproject.org/documents/Science-complete.pdf Visitada sept., 13, 2005.

MACILWAIN, Colin. World Bank backs Third World centers of excellence plan. Nature, 396, 711, p. 24-31. 1998.

MCT (Ministério da Ciência e da Tecnologia). Plano Estratégico do MCT 20042007. 2005. En: <http://ftp.mct.gov.br/sobre/pdf/plano estrategico.pdf $>$ Visitada feb. 20, 22011.

MCT (Ministério da Ciência e da Tecnologia). Relatório analítico Programa de C,T\&I para Nanotecnologia. Coordenação Geral de Micro e Nanotecnologias. 2008. En: http://www.mct.gov.br/upd_blob/0028/28213.pdf Visitada feb., 20, 2011. NanoCeo (Nanotechnology Citizen Engagement Organization). Base de Datos. 2010. En: http://www.nanoceo.net/nanorisks Visitada dic., 20, 2010.

OCDE (Organización para la Cooperación y el Desarrollo Económico). Tecnología, Productividad y Creación de Empleos: Las Mejores Prácticas de Política. 1998. En: <www.oecd.org/dataoecd/39/28/2759012.pdf> Visitada marzo. 20, 2011.

OEA (Organización de Estados Americanos). Reporte Final de la Cuarta Reunión Regular del Comité Inter-Americano de Ciencia y Tecnología (COMCYT), OEA/ Ser.W/XIII.3.4. 2004. En: <http://www.science.oas.org/COMCYT/english/Resolution.htm>. Visitada abril 4, 2006.

OIA-CTS (Observatorio IberoAmericano de la Ciencia, la Tecnología y la Sociedad). La nanotecnología en Iberoamérica. Situación actual y tendencias. Organización de Estados Iberoamericanos. 2008. En:<http://www.oei.es/observatoriocts/index.php?option $=$ com_content\&view $=$ article\&id $=12$ \&ltemid $=3>$ Visitada marzo. 18, 2011.

RUSHTON, Mark ; Záyago, Edgar \& Foladori, Guillermo. Center of Educational Excellence in Nanotechnology: The Proposed World Bank Scientific Millennium Initiatives and Nanotechnogy in Latin America. En: Armando Barrañón. (Org.). New Nanotechnology Developments. Nova Publishers. 2009.

SPIVAK L'Hoste, Ana; HUBERT, Matthieu; FIGUEROA, Santiago y ANDRINI, Leandro. La estructuración de la investigación argentina en nanociencia y nanotecnología: balances y perspectivas. En: Foladori, Záyago \& Invernizzi (comp.). Perspectivas del desarrollo de las nanotecnologías en América Latina. (en prensa). 
TOMA, H. E. Interfaces e organização da pesquisa no Brasil: da Química à Nanotecnologia. Química Nova 28, 48-51. 2005. En: < http://www.scielo.br/pdf/qn/ v28s0/26775.pdf> Visitada feb., 20, 2011.

ZÁYAGO-LAU, Edgar \& FOLADORI, Guillermo. La nanotecnología en México: un desarrollo incierto. Economía, Sociedad y Territorio, no X, v. 32, p. 143-178. 2010.

Recebido em: 08/11/2011

Aceite final: 19/12/2011 


\section{Apéndice: Laboratorios de NT en países seleccionados de AL (hasta ene.} 2011)

\begin{tabular}{|c|c|c|}
\hline Argentina & rasil & México \\
\hline $\begin{array}{l}\text { - } 2002 \text { CINSO (Centro de } \\
\text { Investigación en } \\
\text { Sólidos), CITEDEF - } \\
\text { Ministeriode Defensa, P. } \\
\text { Bs. As. } \\
\text { - 2005. Laboratorio de } \\
\text { Diseño Estratégico para } \\
\text { la Focalización de } \\
\text { Medicamentos, UNQ, P. } \\
\text { Bs. As. } \\
\text { - 2007. Instituto de } \\
\text { Nanociencia y NT, } \\
\text { Centro Atómico } \\
\text { Bariloche, CNEA, P. Río } \\
\text { Negro. (Actualizado en } \\
\text { 2007 y 2009). } \\
\text { - Centro Atómico } \\
\text { Constituyentes, CNEA, } \\
\text { P. Bs. As. }\end{array}$ & 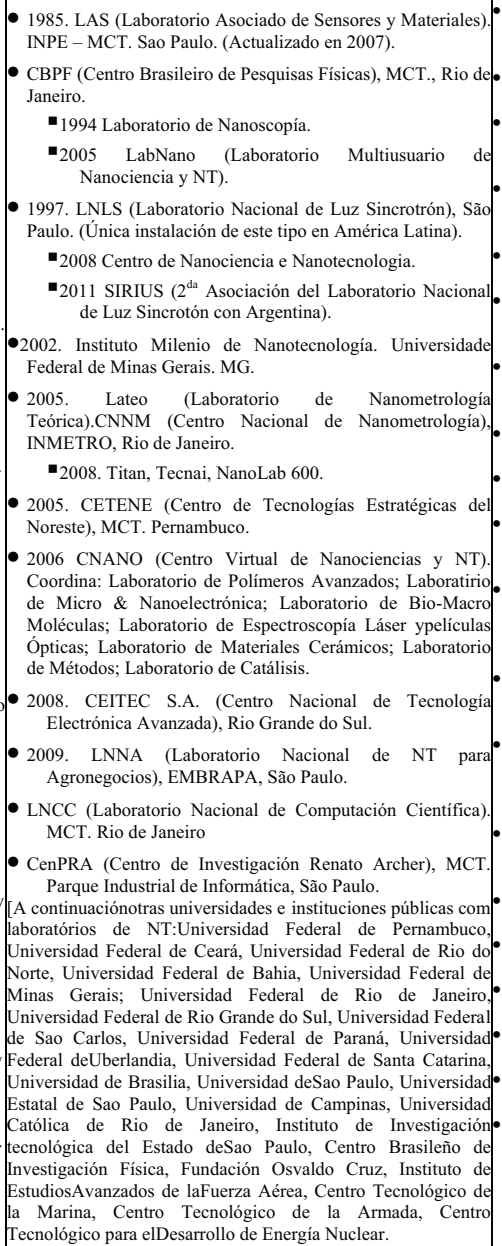 & 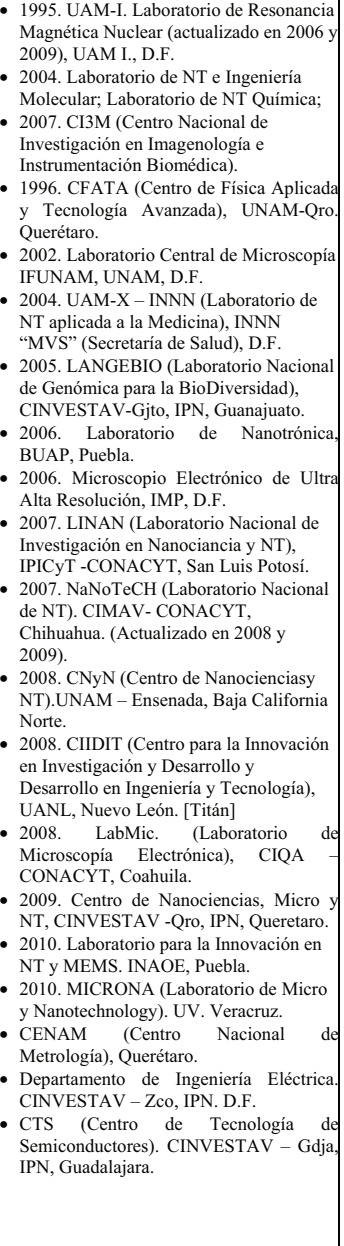 \\
\hline
\end{tabular}

